\title{
Supervised learning of few dirty bosons with variable particle number
}

\author{
P. Mujal ${ }^{1,2^{*}}$, A. Martínez Miguel ${ }^{1,2}$, A. Polls ${ }^{1,2}$, B. Juliá-Díaz ${ }^{1,2}$ and S. Pilati ${ }^{3}$ \\ 1 Departament de Física Quàntica i Astrofísica, Universitat de Barcelona, Martí i Franquès \\ 1, 08028 Barcelona, Spain \\ 2 Institut de Ciències del Cosmos (ICCUB), Universitat de Barcelona, Martí i Franquès 1, \\ 08028 Barcelona, Spain \\ 3 School of Science and Technology, Physics Division, Università di Camerino, 62032 \\ Camerino (MC), Italy \\ * peremujal@gmail.com
}

May 28, 2021

\begin{abstract}
We investigate the supervised machine learning of few interacting bosons in optical speckle disorder via artificial neural networks. The learning curve shows an approximately universal power-law scaling for different particle numbers and for different interaction strengths. We introduce a network architecture that can be trained and tested on heterogeneous datasets including different particle numbers. This network provides accurate predictions for the system sizes included in the training set, and also fair extrapolations to (computationally challenging) larger sizes. Notably, a novel transfer-learning strategy is implemented, whereby the learning of the larger systems is substantially accelerated by including in the training set many small-size instances.
\end{abstract}

\section{Contents}

1 Introduction $\quad 2$

2 Model and Methods 3

2.1 Physical system: few 1D dirty bosons 3

2.2 Network architecture 5

2.3 Training procedure 6

3 Learning the few-body problem

3.1 Homogeneous datasets 7

3.2 Heterogeneous datasets 8

4 Extrapolation and accelerated learning 9

4.1 Extrapolation and accelerated learning for three particles

4.2 Extrapolation and accelerated learning for four particles 11

4.3 Accelerated learning in a real-case scenario 11 


\section{Introduction}

Supervised machine-learning is emerging as a potentially disruptive technique to accurately predict the properties of complex quantum systems. It has already allowed researchers to drastically speed-up various important computational tasks in quantum chemistry and in condensed-matter physics [1, 2], including: molecular dynamics simulations [3 7], electronic structure calculations [8 13], structure-based molecular design [14 16], and protein-molecule binding-affinity predictions $[17,19]$. The deep neural networks represent the most powerful and versatile statistical model. In principle, they can approximate any continuous function with arbitrary accuracy [20]. However, training them without over-fitting requires extremely copious datasets, often comprising hundreds of thousands of training instances. Generating such datasets for large quantum systems is computationally impractical, unless one accepts (sometimes unreliable) approximations such as, e.g., density functional theory. This represents a critical problem that hampers the further development of machine-learning techniques for quantum systems.

A possible approach to circumvent the above problem is to adopt a transfer-learning strategy, as often done in the field of image analysis 21. In the case of quantum systems, transfer learning can be implemented by scaling to larger sizes the neural networks that have been trained on smaller - therefore, computationally tractable - systems. In fact, a form of size scalability is currently being employed in the field of molecular dynamics simulations; in that approach, the ground-state energies are computed as the sum of single-atom contributions, but taking into account only the short-range atomic environments (see, e.g., Ref. [22]). Proper scalability has recently been implemented in a few distinct ways: i) assuming the extensitivity property, using properly constructed size-extensive networks [23]; ii) adopting normalized descriptor-vectors of fixed size (i.e., independent on the physical system size) [24; iii) implementing scalable convolutional networks via global pooling layers, for systems of variable spatial extent [25]. To the best of our knowledge, statistical models that accept the particle number as an explicit system descriptor have not been investigated yet.

In this article, we consider the supervised learning of interacting bosons in a one-dimensional random external field. Our main goals are to quantify the learning speed [26], in terms of prediction accuracy versus number of instances in the training set, and to implement flexible neural networks that can address different particle numbers simultaneously. The Hamiltonian we focus on is realistic and describes experiments performed with ultracold atoms in optical speckle fields [27]. It represents a challenging computational task, belonging to the family of dirty boson problems [28]. Recently, this model has been addressed in a study on the stability of the Anderson localization phenomenon against inter-particle interaction 29]. The model was shown to host a many-body localized phase. Here, we analyse how many instances are needed to train deep neural networks to accurately predict its ground-state energy, depending on the interaction strength and on the particle number. The training and the test sets are 
produced via an exact diagonalization technique. This choice allows us to avoid the common approximations employed in most supervised-machine learning studies. However, it limits our analysis to small particle numbers, specifically, up to four bosons.

Notably, we implement a neural network for continuous-space quantum systems with variable particle number. This network combines the scalable convolutional architecture of Ref. 25] [see Fig. 1, panel (b)], which can address disordered systems of variable spatial extent (but fixed particle number), with an additional descriptor representing the particle number. This descriptor bypasses the convolutional layers and is fed directly to the final dense layers [see Fig. 1. panel (a)]. As we demonstrate here, this network is able to accurately predict the ground-state energies of systems with different particle numbers, even when considering heterogeneous datasets including instances with different size. The learning speed appears to be independent on the particle number and on the interaction strength. In fact, the prediction accuracy follows an approximately universal power-law scaling with the number of instances in the training set. Our neural network can also be used to perform fairly accurate extrapolations to particle numbers larger than those included in the training set. Furthermore, we show that the learning of the larger sizes can be substantially accelerated if a training set with many small-size instances, which are computationally accessible, is augmented with a small amount of instances for the larger particle number. This strategy provides accurate predictions also for the larger size with a computationally feasible training set. It represents an alternative transfer-learning technique, paving the way to a novel approach to accurately predict the properties of complex quantum systems, for which copious training sets cannot be generated in feasible computational times.

Our study begins with an analysis of the learning speed, considering both datasets with a unique particle number, as well as the combined learning with heterogeneous datasets. Then, we analyse the accuracy of the extrapolations to particle numbers larger than those included in the training set, as well as the accelerated learning of relatively large systems using data for smaller sizes. In detail, the rest of the article is organized as follows: the physical system we address and the computational method we employ to determine its ground-state energy are provided in Section 2, together with a description of the artificial neural network introduced in this article and some details on the training algorithm. The analysis on the learning speed of the few-boson problem is reported in Section 3 . Section 4 reports the analysis on the extrapolation procedure and on the accelerated learning. The summary of our main findings and some future perspectives are reported in Section 5.

\section{Model and Methods}

\subsection{Physical system: few 1D dirty bosons}

We consider a one-dimensional system of few repulsively interacting bosons in the presence of an external disordered potential. This model has been experimentally engineered and describes ultracold atoms subjected to optical speckle fields and confined in cigar-shaped traps. Specifically, it corresponds to the setup of early cold-atom experiments on the Anderson localization phenomenon [30,31]. The Hamiltonian of the system reads

$$
\mathcal{H}=\sum_{i=1}^{N}\left(-\frac{\hbar^{2}}{2 m} \frac{\partial^{2}}{\partial x_{i}^{2}}+V\left(x_{i}\right)\right)+\sum_{i<j}^{N} v\left(x_{i}, x_{j}\right)
$$




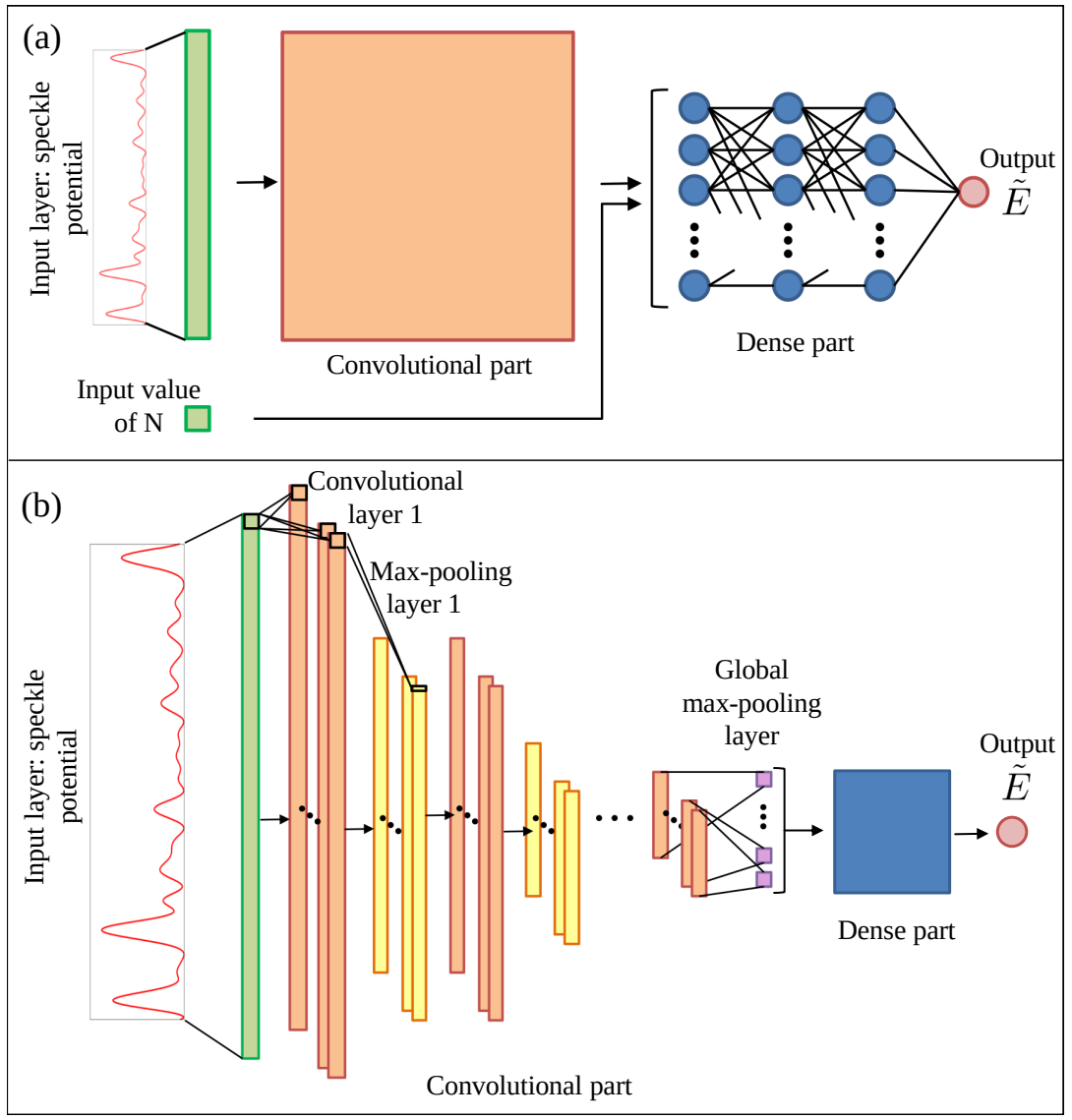

Figure 1: (a) Schematic representation of the deep feed-forward neural network used to predict the ground-state energy, $\tilde{E}$, of few-boson systems (output). The input descriptors are the values of the speckle potential on a fine discrete grid. In the case of training with heterogeneous datasets, an additional system descriptor is included, representing the particle number $N$. This descriptor is connected directly to the dense part of the network. (b) Structure of the convolutional part of the neural network. This model is used when training on homogeneous datasets including instances with a unique particle number.

where $m$ is the particle mass, $N$ the number of particles, and $x_{i}$ corresponds to the position of particle $i$, with $i=1, \ldots, N$. The two-body interaction is described by a contact interaction potential,

$$
v\left(x_{i}, x_{j}\right)=g \delta\left(\left|x_{i}-x_{j}\right|\right),
$$

where $g$ is the parameter that defines the interaction strength. Its sign determines the character of the interaction: repulsive for $g>0$ and attractive for $g<0$. We focus on the repulsive case.

The external potential $V(x)$ represents the effect of optical speckle fields on ultracold atoms. It can be generated on a discrete spatial grid with fine spacing via the stochastic numerical algorithm described in detail in Refs. 32, 33. We produce many instances of speckle potentials using different pseudo-random numbers. All instances are characterized by the same spatial correlation length, indicated in the following as $\ell$, and by the same average intensity $V_{0}$. The correlation length allows one to define a characteristic energy scale, namely, 


\begin{tabular}{|l|l|l|}
\hline Layer name & Layer Function & Layer description \\
\hline Input layer 1 & Input & $\begin{array}{l}\text { The value of the speckle potential in 1024 } \\
\text { points }\end{array}$ \\
\hline Convolutional layers & ReLu & $\begin{array}{l}50 \quad \text { filters, kernel_size=5, strides=1, } \\
\text { padding=same, activation=relu }\end{array}$ \\
\hline $\begin{array}{l}\text { Local max-pooling lay- } \\
\text { ers }\end{array}$ & Max-pooling & Local pooling with pool_size=3 \\
\hline $\begin{array}{l}\text { Global max-pooling } \\
\text { layer }\end{array}$ & Global max-pooling & $\begin{array}{l}50 \text { neurons = number of filters in the pre- } \\
\text { ceding convolutional layer }\end{array}$ \\
\hline Input layer 2 & Input & $N$, number of particles \\
\hline Dense layers & ReLu & 30 neurons, activation=relu \\
\hline Output layer & Identity & 1 neuron, activation=identity \\
\hline
\end{tabular}

Table 1: Details of the layers constituting the convolutional and the dense parts of our neural network. Definitions are standard, see for instance Ref. [37].

the correlation energy $E_{c}=\hbar^{2} /\left(m \ell^{2}\right)$. In the following, we consider speckle fields of fixed spatial extent, namely, $L=20 \ell$, with hard-wall boundary conditions. The spatial grid for the speckle potential includes 1024 points, corresponding to a grid spacing $\delta x \simeq 0.153 \ell$. With such a fine grid, discretization effects are negligible. The disorder strength is fixed at $V_{0}=5 E_{c}$. Different values of the interaction parameter $g$ are considered; they are expressed in the following in units of $\hbar^{2} /(\ell m)$. Specifically, from now on we consider the weak interaction $g=0.05$, an intermediate value $g=0.26$, and the strong-coupling case $g=1$.

We train deep neural networks to predict the ground-state energies of different instances of the Hamiltonian (1). These energies are computed by means of the exact-diagonalization method described in Ref. [29]. This method is based on a second-quantization formalism. The Fock space of the $N$ bosons is built using the basis of the single-particle eigenstates of the kinetic energy operator. The diagonalization is performed in a truncated space including only the Fock basis states with kinetic energy smaller than a chosen threshold, following the technique introduced in Ref. [34]. This energy threshold determines both the dimension of the truncated $N$-boson Fock space $D_{\mathrm{MB}}$, and the required number of single-particle basis states $M$. Further details on the computational technique we employ are reported in Ref. [35]. The energy thresholds we adopt in this article lead to the following truncation parameters: for $N=1$, we have $M=D_{\mathrm{MB}}=100$; for $N=2, M=100$ and $D_{\mathrm{MB}}=3914$; for $N=3, M=100$ and $D_{\mathrm{MB}}=88106$; and for $N=4, M=80$ and $D_{\mathrm{MB}}=552099$. The computational resources available to us allow producing datasets including different numbers of instances; specifically, we produce $600000,50000,2000$, and 270 instances for $N=1,2,3$, and 4 , respectively, for each of the three values of the interaction parameter $g$ we consider. These datasets are available at $[36]$.

\subsection{Network architecture}

In Ref. [25], deep feed-forward neural networks have been employed in the supervised learning of the ground-state energy of the Hamiltonian (1). However, that study addressed only the single particle case, namely, the case $N=1$. A scalable architecture was implemented using standard convolutional layers connected to dense hidden layers (i.e., with all-to-all 
connectivity) via a global pooling operation. This allows the model to address disordered systems of arbitrary spatial extent $L$. Our goal is to further develop that architecture so that it can address also an arbitrary particle number $N$.

Our investigation first addresses homogeneous datasets including instances with a single particle number, either $N=1, N=2$, or $N=3$. For this purpose, the architecture of Ref. [25] (represented in panel (b) of Fig. 1) can be employed without modifications. The system instances are represented by 1024 descriptors corresponding to the speckle potential intensities $V\left(x_{k}\right)$ on the spatial grid $x_{k}=k \delta x$, with $k=0, \ldots, 1023$. Since the grid spacing $\delta x$ is much smaller than the disorder correlation length $\ell$, these 1024 descriptors provide an exhaustive representation of the speckle potential of each instance. The 1024 descriptors are fed to the convolutional part of the architecture. This part includes six convolutional layers with 50 filters, each followed by a local pooling layer. The output of the convolutional part is forwarded to the first of three dense layers, each including 30 neurons, via a global pooling layer. The final layer includes a single neuron. Its activation should correspond to the ground-state energy. Thanks to the global pooling layer, this architecture can be applied to systems with different spatial extent $L$ (and, hence, different numbers of descriptors), without re-training.

To address heterogeneous datasets containing instances with different particle numbers, we have to extend the architecture shown in panel (b) of Fig. 1. Specifically, we include an additional descriptor whose value corresponds to the particle number $N$. The corresponding neuron is linked directly to the first dense layer, bypassing the convolutional and the pooling layers (see panel (a) of Fig. 1). In principle, this should allow the model to learn how the ground-state energy depends on the particle number, providing predictions for arbitrary $N$. In Sections 3 and 4 we quantify if and to what extent this goal is achieved. All details of the neural-network structure are reported in Table 1 .

\subsection{Training procedure}

The training is performed by minimizing the mean squared error (MSE), defined as:

$$
\mathrm{MSE}=\frac{1}{N_{\text {train }}} \sum_{t=1}^{N_{\text {train }}}\left(\tilde{E}_{t}-E_{t}\right)^{2}
$$

where $E_{t}$ and $\tilde{E}_{t}$ are, respectively, the exact and the predicted ground-state energies of the training instance $t . N_{\text {train }}$ is the number of instances included in the training set. The optimization of the neural-network weights and biases is performed using the Adam algorithm [38], as implemented in the Keras python library [39. An early stopping criterion is adopted. It is based on the MSE of a validation set (distinct from the training set). The optimal network parameters obtained throughout the training process are retained. To quantify the accuracy of the predictions provided by the trained networks we consider two figures of merit. The first is the mean absolute error (MAE), defined as:

$$
\mathrm{MAE}=\frac{1}{N_{\text {test }}} \sum_{t=1}^{N_{\text {test }}}\left|\tilde{E}_{t}-E_{t}\right| .
$$


The second is the coefficient of determination, defined as:

$$
\mathrm{R}^{2}=1-\frac{\sum_{t=1}^{N_{\text {test }}}\left(\tilde{E}_{t}-E_{t}\right)^{2}}{\sum_{t=1}^{N_{\text {test }}}\left(E_{t}-\langle E\rangle\right)^{2}} .
$$

Here, $N_{\text {test }}$ is the number of instances in the test set, and $\langle E\rangle$ is their average ground-state energy. We stress that the instances included in the test set are distinct from those used for training and for validation. It is worth recalling that perfect predictions correspond to the score $R^{2}=1$, while a constant function predicting the correct average $\langle E\rangle$ corresponds to the score $R^{2}=0$. For the results reported in the following sections, unless otherwise specified, $20 \%$ of the datasets are used for testing. The remaining $80 \%$ is divided into the training data, accounting a $75 \%$, and validation data, corresponding to the remaining $25 \%$. It is worth mentioning that in our analysis we do not employ regularization techniques since the MAE on the training and validation sets are very close. For the smallest training sets the performance might be slightly improved by using regularization terms.

\section{Learning the few-body problem}

\subsection{Homogeneous datasets}

The neural networks described in the previous section are trained to predict the ground-state energy of the Hamiltonian (1). We first analyse homogeneous datasets including instances with a unique particle number. In this first analysis, the networks are trained and tested on the same system size, considering the cases $N=1, N=2$, and $N=3$ separately. Since in this analysis the particle number is fixed, we adopt the network architecture shown in panel (b) of Fig. 2, i.e., the one that only accepts external potential values as system descriptors. Three values of the interaction parameter are (separately) considered, namely, $g=0.05, g=0.26$, and $g=1$. The first choice corresponds to the weakly-interacting regime, where the ground-state energies are not far from their non-interacting values. The second choice represents an intermediate interaction strength, and the third choice is close to the Tonks-Girardeau limit where the bosonic ground-state energy approaches the result corresponding to (non-interacting) identical fermions. The learning speed is analysed in Fig. 2 . The prediction accuracy, as measured by the MAE per particle computed on the test set, is plotted as a function of the number of instances included in the training set $N_{\text {train. }}$. It is worth reminding that the test is performed on instances not included in the training and the validation sets. In general, one expects a power-law scaling of the prediction accuracy, corresponding to MAE $/ N \propto N_{\text {train }}^{-b}$ [40, 41], where $b>0$. Interestingly, we find that the data for all particle numbers and for all interaction strengths we consider are consistent with a power-law scaling with the same exponent $b=0.5$ (see dashed line in Fig. 2). These results suggest an approximate universal behavior, at least for the one-dimensional manybody localized model we address. While our focus is on the scaling exponent $b$, one notices that the datasets corresponding to different $N$ and $g$ essentially overlap, within the statistical uncertainties. This suggests that also the prefactor is, at least approximately, universal. 


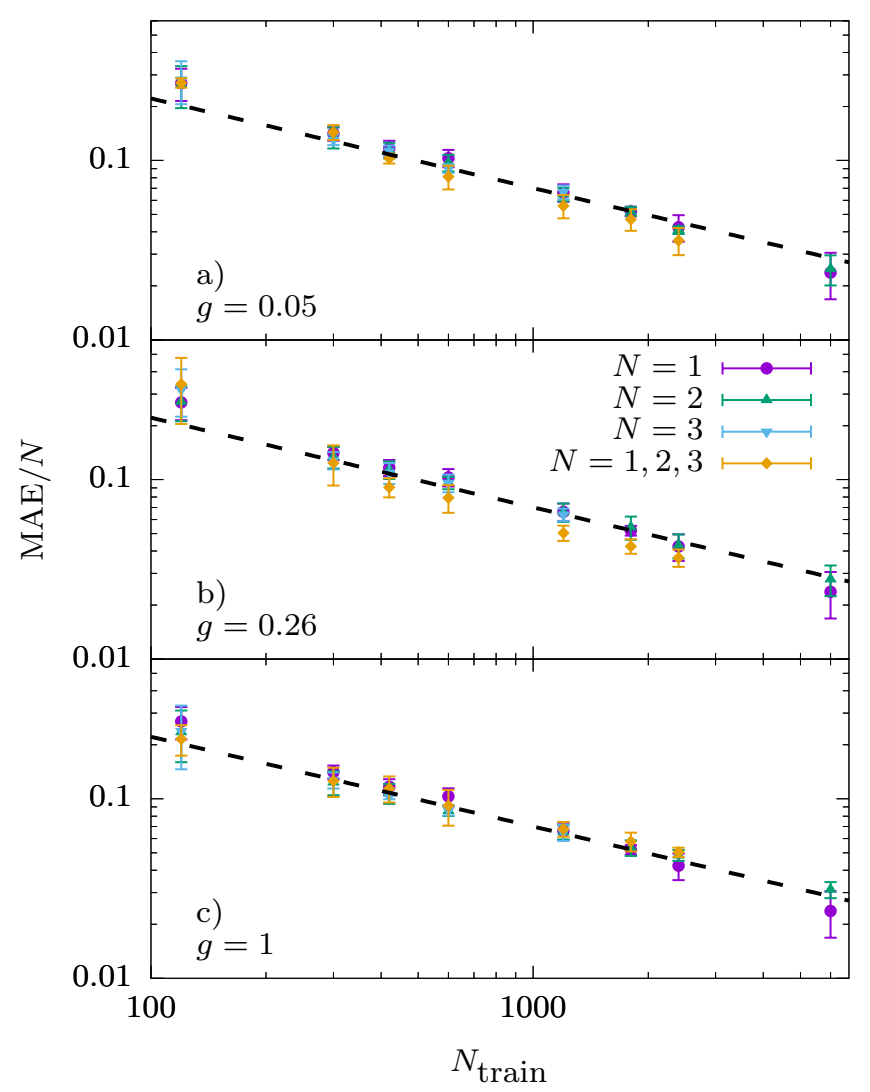

Figure 2: Mean absolute error per particle $\mathrm{MAE} / N$, computed on the test ground-state energies, as a function of the number of instances in the training set $N_{\text {train }}$. The different symbols correspond to training on homogeneous datasets including a unique particle number (either $N=1, N=2$, or $N=3$ ) and to combined training and testing on heterogeneous datasets including all three particle numbers $(N=1,2,3)$. The three panels correspond to different interaction strengths $g$. The errorbar is the estimated standard deviation of the mean obtained with up to eight independent models trained with different pseudo-random numbers. The dashed line corresponds to a power-law scaling with $b=0.5$, see text for details.

\subsection{Heterogeneous datasets}

One of our main goals is to implement models that can address different system sizes simultaneously. This is achieved via the modified neural-network shown in panel (a) of Fig. 1. This model is fed with an additional descriptor representing the particle number $N$, beyond the 1024 speckle potential intensities. We train and test this model using heterogeneous datasets which include system instances with different particle numbers, with equal populations for the three $N$ values. As before, training and testing are performed for the same interaction parameter, addressing separately the three values we consider. We stress, however, that in this case the same neural network predicts ground-state energies for different particle numbers, while in the previous analysis different models were employed for each case. Notably, the MAE per particle follows the same power-law scaling with exponent $b=0.5$, as previously 


\begin{tabular}{|c|c|c|c|c|c|c|}
\hline & $g=0.05$ & $g=0.26$ & \multicolumn{2}{c|}{$g=1$} \\
\hline & $R^{2}$ & MAE & $R^{2}$ & MAE & $R^{2}$ & MAE \\
\hline \multicolumn{7}{|c|}{ Trained with $N=1,2$} \\
\hline$N=1(1800)$ & 0.992 & 0.027 & 0.991 & 0.027 & 0.987 & 0.035 \\
\hline$N=2(1800)$ & 0.994 & 0.047 & 0.995 & 0.042 & 0.988 & 0.065 \\
\hline$N=3$ (Extrap.) & 0.912 & 0.299 & 0.880 & 0.366 & 0.848 & 0.374 \\
\hline \multicolumn{7}{|c|}{ Accelerated learning for $N=3$} \\
\hline$N=1(1800)$ & 0.993 & 0.024 & 0.991 & 0.029 & 0.987 & 0.037 \\
\hline$N=2(1800)$ & 0.992 & 0.046 & 0.994 & 0.045 & 0.991 & 0.057 \\
\hline$N=3(200)$ & 0.992 & 0.076 & 0.993 & 0.080 & 0.984 & 0.120 \\
\hline \hline \multicolumn{7}{|c|}{ Trained with $N=1,2,3$} \\
\hline$N=1(1200)$ & 0.993 & 0.026 & 0.987 & 0.039 & 0.980 & 0.042 \\
\hline$N=2(1200)$ & 0.991 & 0.050 & 0.991 & 0.058 & 0.992 & 0.054 \\
\hline$N=3(1200)$ & 0.995 & 0.065 & 0.995 & 0.070 & 0.995 & 0.065 \\
\hline$N=4$ (Extrap.) & 0.977 & 0.172 & 0.920 & 0.313 & 0.830 & 0.481 \\
\hline \multicolumn{7}{|c|}{ Accelerated learning for $N=4$} \\
\hline$N=1(1200)$ & 0.987 & 0.037 & 0.981 & 0.046 & 0.980 & 0.049 \\
\hline$N=2(1200)$ & 0.987 & 0.068 & 0.988 & 0.068 & 0.987 & 0.069 \\
\hline$N=3(1200)$ & 0.991 & 0.093 & 0.990 & 0.099 & 0.990 & 0.099 \\
\hline$N=4(200)$ & 0.983 & 0.160 & 0.984 & 0.148 & 0.988 & 0.124 \\
\hline
\end{tabular}

Table 2: Performance of the neural network in the test-case considered in Figs. 3 and 4 . The coefficient of determination $R^{2}$ and the mean absolute error MAE are reported for three interaction strengths $g$, considering networks trained on $N=1,2$ and on $N=1,2,3$. The test results are shown for the particle numbers $N$ included in the training set (number of training instances in parenthesis), for the extrapolations to $N=3$ and to $N=4$, and for the accelerated learning with additional large-size instances in the training sets.

found in the analysis with separate particle numbers. This further supports the statement about an approximately universal behaviour.

\section{Extrapolation and accelerated learning}

The computational cost required to solve many-body problems increases exponentially fast with the number of particles. For example, with our exact-diagonalization technique the cost increases by a factor $\approx 27$ going from the $N=2$ case to the $N=3$ case, as well as when going from the $N=3$ to the $N=4$ case. Hence, one expects that the datasets one encounters in practical scenarios contain many small $N$ instances, and only very few instances for relatively large $N$. It is therefore natural to wonder (i) if a variable- $N$ neural network can perform extrapolations, providing predictions for system sizes larger than those included in the training sets, and (ii) if the many small- $N$ instances can be used to accelerate the training process for larger $N$, enabling the network to provide accurate predictions even when only very few training instances are available for the larger system size. In the following, we address these relevant issues using the variable- $N$ architecture shown in the panel (a) of Fig. 1. First, in Section 4.1 we focus on the extrapolation and on the accelerated learning of 


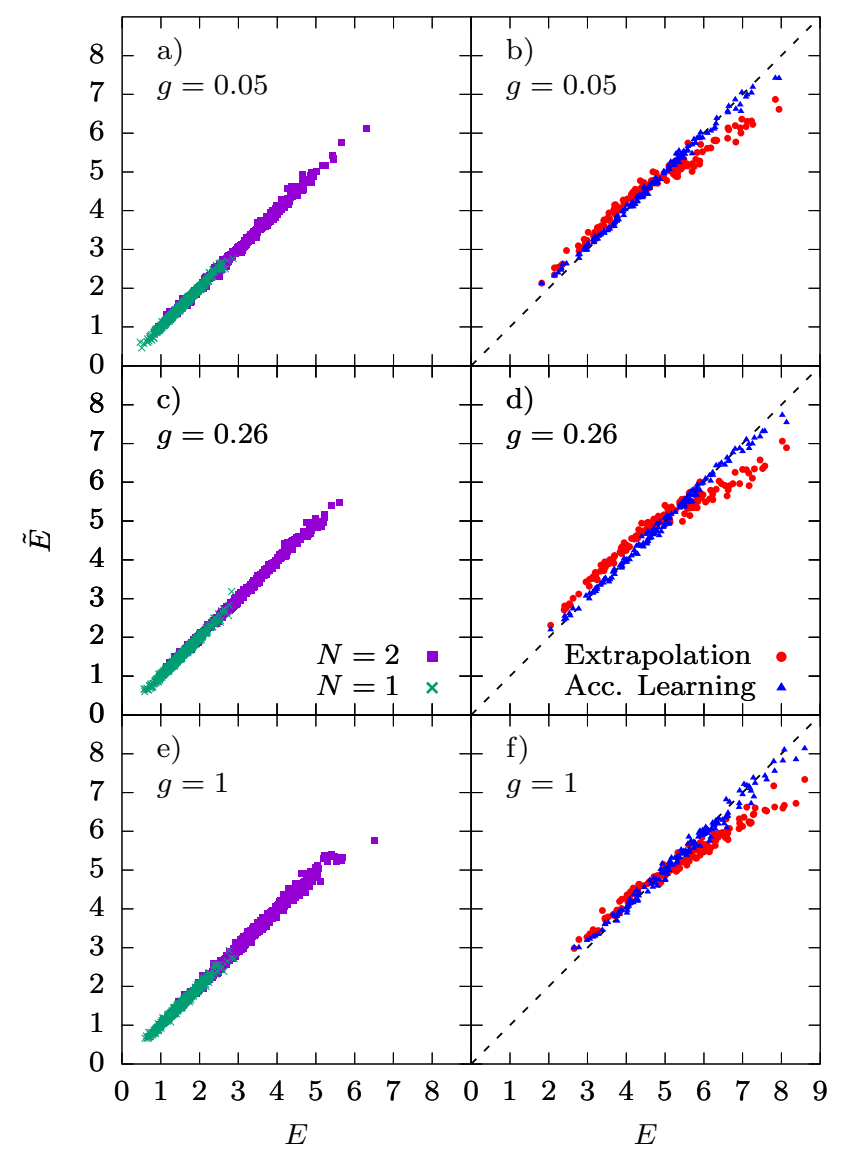

Figure 3: Ground-state energies $\tilde{E}$, predicted by a neural network trained on a heterogeneous dataset, as a function of the exact-diagonalization $E$. The training sets include 1800 instances for $N=1$ and as many for $N=2$. Panels (a), (c), and (e) report results for the systems sizes included in the training set. Panels (b), (d), and (f) report the extrapolations to the $N=3$ case, and the accelerated learning with 200 additional instances for $N=3$. The three rows correspond to different interaction strengths $g$.

the $N=3$ case, using data for $N=1$ and $N=2$; then, in Section 4.2 we address the $N=4$ case, where we use data for $N=1,2$, and 3. Finally, in Section 4.3 we consider a real-case scenario with much larger databases for lower particle numbers.

\subsection{Extrapolation and accelerated learning for three particles}

In the first case, a network is trained on a dataset including 1800 instances for $N=1$ and as many for $N=2$. This network is then used to predict the ground-state energies of $N=3$ instances. To quantify the prediction accuracy we consider the MAE and the coefficient of determination $R^{2}$. The corresponding values are reported in Table 2 For the system sizes included in the training set, namely, $N=1$ and $N=2$, the predictions are extremely accurate, 
corresponding to $R^{2} \gtrsim 0.99$. The high degree of accuracy can be appreciated also in the scatter plots of Fig. 3 (left panels), where the predicted energies for the test set are plotted as a function of the exact-diagonalization results. Interestingly, also the extrapolation to $N=3$ are fairly accurate, providing coefficients of determination $R^{2} \gtrsim 0.85$ for all interaction strengths. The predictions appear to deviate from the exact values mostly in the large energy regime (see right panels of Fig. 3). The MAE per particle is around MAE/N $\simeq 0.1$. While this accuracy is remarkable, given that no $N=3$ instance is exploited in the training process, it might not be sufficient for practical applications of supervised machine learning. Hence, we analyse the effect of adding to the previous training set just 200 instances for the particle number $N=3$. Interestingly, the combined training with the $N=1, N=2$, and $N=3$ instances leads to high accuracy for all three system sizes. The coefficient of determination is $R^{2} \gtrsim 0.99$. The MAE per particle for $N=3$ is MAE $/ N \simeq 0.03$, i.e., close to the accuracy obtained for $N=1$ and for $N=2$. Notably, the performances on the two smaller system sizes do not degrade. For the sake of comparison, it is worth noticing that, when the network is trained using only $N=3$ instances (see Section 3), the MAE per particle reached with just 200 training instances is approximately an order of magnitude larger. These results indicate that the combined training with smaller sizes provides a boost to the learning process for the larger size, allowing the network to reach high accuracy with fewer training instances.

\subsection{Extrapolation and accelerated learning for four particles}

The procedure described above is now extended to $N=4$ systems. First, a network trained on a dataset including 1200 instances for $N=1$, as many for $N=2$ as well as for $N=3$ (corresponding to a total of 3600 instances), is used to predict the ground-state energies of $N=4$ instances. The scatter plots of these extrapolations are shown in the right panels of Fig. 4. The prediction accuracy is remarkable, considering that no $N=4$ instance is used in the training process. The coefficient of determinations are: $R^{2} \simeq 0.97$ for the weakly interacting case $g=0.05, R^{2} \simeq 0.92$ for $g=0.26$, and $R^{2} \simeq 0.84$ for the strongly interacting case $g=1$. This indicates that the network is learning how the ground-state energy scales with the particle number, at least for the weak and the intermediate interactions. Next, we test the efficiency of accelerated learning. We include in the previous training set 200 instances for $N=4$. Again, we find remarkably accurate results, corresponding to a coefficient of determination $R^{2} \gtrsim 0.98$ and a MAE/ $N \sim 0.035$ for all interaction strengths. For comparison, a network trained only on $200 N=4$ instances (using the model of panel (b) of Fig. 1) would reach MAE $/ N \sim 0.17\left(R^{2} \simeq 0.7\right)$. Again, this indicates that transfer learning from smaller to larger system sizes is effective, allowing one to accelerate the training process for the larger systems.

\subsection{Accelerated learning in a real-case scenario}

Since the computational cost of solving many-body instances increases exponentially fast with the systems size, in practical applications of supervised learning the training sets inevitably contain significantly fewer instances for the larger particle numbers. Here, we analyse the efficiency of the accelerated learning with the typical training dataset one would encounter in a real-case scenario. Specifically, this dataset includes, 360000 instances for $N=1,30000$ for $N=2,1200$ for $N=3$, and 200 for $N=4$. It is worth noticing that more computational time is invested in the larger particle numbers, since one expects that larger systems provide 


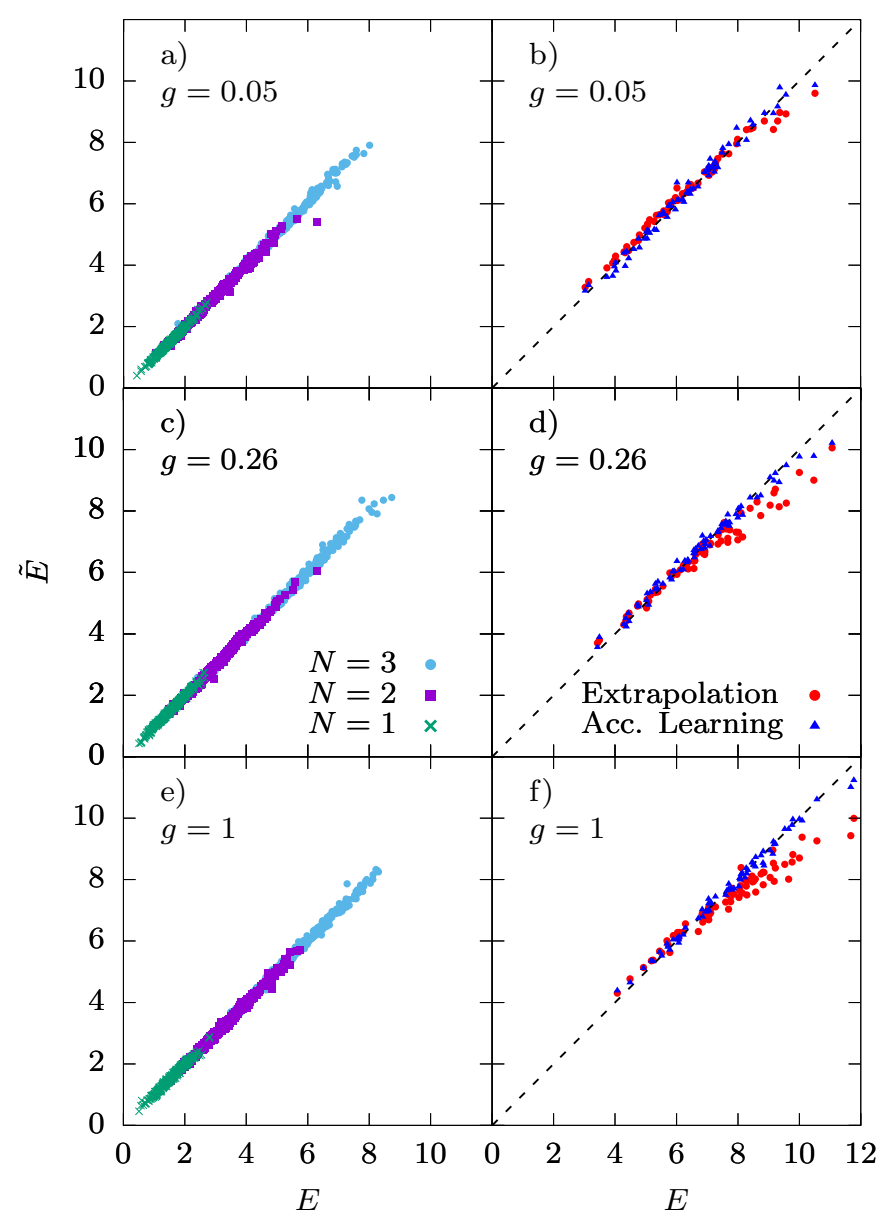

Figure 4: Ground-state energies $\tilde{E}$, predicted by neural networks trained on heterogeneous datasets, as a function of the exact-diagonalization results $E$. The training sets include 1200 instances for the particle numbers $N=1,2$, and 3, for a total of 3600 instances. Panels (a), (c), and (e) report results for the systems sizes included in the training set. Panels (b), (d), and (f) report the extrapolations to the $N=4$ case, and the accelerated learning with 200 additional training instances for $N=4$. The three rows correspond to different interaction strengths $g$.

more information about the scaling of the ground-state properties with the system size. The performance of the extrapolations and of the accelerated learning is summarized in Table 3 . where we report the MAE and the coefficient of determination. Interestingly, the extrapolations are significantly more accurate than those reported in Section 4.2, which were based on fewer training instances (see Table 2). In particular, the extrapolations to $N=4$ (based on training instances for $N=1, N=2$, and $N=3$ ) reach $R^{2} \gtrsim 0.97$. This indicates that, when the training set includes a sufficient number of training instances for relatively small system sizes, the network can learn to accurately scale the predictions to larger particle numbers. 


\begin{tabular}{||c|c|c|c|c|c|c||}
\hline & \multicolumn{2}{|c|}{$g=0.05$} & \multicolumn{2}{c|}{$g=0.26$} & \multicolumn{2}{c||}{$g=1$} \\
\hline & $R^{2}$ & MAE & $R^{2}$ & MAE & $R^{2}$ & MAE \\
\hline \multicolumn{7}{|c|}{ Trained with $N=1,2$} \\
\hline$N=1(360000)$ & 0.9994 & 0.0078 & 0.9994 & 0.0076 & 0.9992 & 0.0082 \\
\hline$N=2(30000)$ & 0.9994 & 0.0138 & 0.9986 & 0.0231 & 0.9975 & 0.0298 \\
\hline$N=3$ (Extrap.) & 0.9641 & 0.1889 & 0.9335 & 0.3049 & 0.9445 & 0.2280 \\
\hline \multicolumn{7}{|c|}{ Accelerated learning for $N=3$} \\
\hline$N=1(360000)$ & 0.9996 & 0.0063 & 0.9993 & 0.0081 & 0.9993 & 0.0081 \\
\hline$N=2(30000)$ & 0.9996 & 0.0123 & 0.9986 & 0.0223 & 0.9978 & 0.0277 \\
\hline$N=3(1500)$ & 0.9994 & 0.0219 & 0.9968 & 0.0552 & 0.9928 & 0.0798 \\
\hline \hline \multicolumn{7}{|c|}{ Trained with $N=1,2,3$} \\
\hline$N=1(360000)$ & 0.9995 & 0.0069 & 0.9993 & 0.0081 & 0.9987 & 0.0111 \\
\hline$N=2(30000)$ & 0.9995 & 0.0138 & 0.9989 & 0.0212 & 0.9952 & 0.0418 \\
\hline$N=3(1200)$ & 0.9993 & 0.0234 & 0.9975 & 0.0534 & 0.9904 & 0.0918 \\
\hline$N=4($ Extrap.) & 0.9934 & 0.1140 & 0.9890 & 0.1385 & 0.9777 & 0.1935 \\
\hline \multicolumn{7}{|c|}{ Accelerated learning for $N=4$} \\
\hline$N=1(360000)$ & 0.9994 & 0.0074 & 0.9994 & 0.0075 & 0.9992 & 0.0087 \\
\hline$N=2(30000)$ & 0.9992 & 0.0165 & 0.9987 & 0.0226 & 0.9974 & 0.0301 \\
\hline$N=3(1200)$ & 0.9988 & 0.0346 & 0.9974 & 0.0523 & 0.9938 & 0.0789 \\
\hline$N=4(200)$ & 0.9987 & 0.0438 & 0.9925 & 0.1100 & 0.9882 & 0.1459 \\
\hline
\end{tabular}

Table 3: Performance of the neural network in the real-case scenario. The coefficient of determination $R^{2}$ and the mean absolute error MAE are reported for three interaction strengths $g$, considering networks trained on $N=1,2$ and on $N=1,2,3$. The test results are shown for the particle numbers $N$ included in the training set (number of training instances in parenthesis), for the extrapolations to $N=3$ and to $N=4$, and for the accelerated learning.

Furthermore, 200 instances for $N=4$ are sufficient to further improve the accuracy. Again, this indicates that the network is capable of transfer the knowledge acquired on smaller system sizes, using it to drastically accelerate the learning of larger-system properties.

\section{$5 \quad$ Summary and conclusions}

We have addressed the supervised learning of the (few) dirty boson problem, considering a specific Hammiltonian which has been already implemented in cold-atom experiments. The training and the test sets have been produced via an exact diagonalization technique, avoiding the uncontrolled approximations often employed in analogous studies on the supervised learning of quantum systems. This limited our analysis to relatively small systems, specifically, up to four bosons. These datasets are made publicly available at Ref. [36] to support future comparative studies on the supervised training of deep neural networks. Our findings indicate that the learning curve, in terms of accuracy of ground-state energy predictions versus number of training instances, is approximately universal for different particle numbers and for different interaction strengths.

The artificial neural network we introduced can be trained and tested on heterogeneous 
datasets including instances with different particle numbers. This is achieved by combining a convolutional architecture which can address disordered fields of variable spatial extent, with an additional descriptor that explicitly represents the particle number. This descriptor is fed to the final dense layers, bypassing the convolutional part. This detail constitutes a relevant innovative aspect of our architecture. Our analysis demonstrates that this network provides accurate ground-state energy predictions, independently of the particle number, at least within the system sizes we considered. Furthermore, it allows one to perform extrapolations to particle numbers larger than those included in the training set. Notably, the learning of relatively large systems can be accelerated using heterogeneous training sets including many small-size instances and only a small amount of large-size instances. This represents the typical scenario, given the rapidly growing computational cost of solving quantum models. This strategy is somewhat analogous to the transfer learning protocols commonly employed in the field of image analysis, whereby deep neural networks pre-trained on large datasets relevant examples are the ResNet [42] and the VGG models [43] - are then specialized on the desired classification task using much smaller samples. Here we implemented transfer learning from small to larger particle numbers using heterogeneous datasets.

In future work, it would be interesting to further explore the universality of the learning curve, considering setups with different models of disorder, interatomic potentials, geometries, or particle statistics. Furthermore, it would be important to extend our analysis to larger particle numbers, possibly in combination with different computational techniques, such as, e.g., quantum Monte Carlo simulations. As a future perspective, one can envision the use of coldatom experiments as quantum simulators to produce the datasets required to train neural network for computationally intractable models. We argue that flexible neural-network architecture and transfer learning strategies shall play a critical role in the practical applications of cold-atom quantum simulators.

\section{Acknowledgements}

We acknowledge useful discussions with S. Cantori, A. Dauphin, F. Isaule and I. Morera.

Funding information S. P. acknowledges financial support from the FAR2018 project titled "Supervised machine learning for quantum matter and computational docking" of the University of Camerino and from the Italian MIUR under the project PRIN2017 CEnTraL 20172H2SC4. This work has been partially supported by MINECO (Spain) Grant No. FIS2017-87534-P. We acknowledge financial support from Secretaria d'Universitats i Recerca del Departament d'Empresa i Coneixement de la Generalitat de Catalunya, co-funded by the European Union Regional Development Fund within the ERDF Operational Program of Catalunya (project QuantumCat, ref. 001-P-001644). S. P. also acknowledges the CINECA award under the ISCRA initiative, for the availability of high performance computing resources and support. 


\section{References}

[1] G. Carleo, I. Cirac, K. Cranmer, L. Daudet, M. Schuld, N. Tishby, L. Vogt-Maranto and L. Zdeborová, Machine learning and the physical sciences, Rev. Mod. Phys. 91(4), 045002 (2019), doi:10.1103/RevModPhys.91.045002.

[2] J. Carrasquilla, Machine learning for quantum matter, Advances in Physics: X 5(1), 1797528 (2020), doi:10.1080/23746149.2020.1797528.

[3] T. B. Blank, S. D. Brown, A. W. Calhoun and D. J. Doren, Neural network models of potential energy surfaces, J. Chem. Phys. 103(10), 4129 (1995), doi:10.1063/1.469597.

[4] J. Behler and M. Parrinello, Generalized neural-network representation of highdimensional potential-energy surfaces, Phys. Rev. Lett. 98(14), 146401 (2007), doi:10.1103/PhysRevLett.98.146401.

[5] J. Behler, Neural network potential-energy surfaces in chemistry: a tool for large-scale simulations, Phys. Chem. Chem. Phys. 13(40), 17930 (2011), doi:10.1039/C1CP21668F.

[6] A. P. Bartók, S. De, C. Poelking, N. Bernstein, J. R. Kermode, G. Csányi and M. Ceriotti, Machine learning unifies the modeling of materials and molecules, Sci. Adv. 3(12), e1701816 (2017), doi:10.1126/sciadv.1701816.

[7] L. Zhang, J. Han, H. Wang, W. Saidi, R. Car and E. Weinan, End-to-end symmetry preserving inter-atomic potential energy model for finite and extended systems, In S. Bengio, H. Wallach, H. Larochelle, K. Grauman, N. Cesa-Bianchi and R. Garnett, eds., Adv. Neural Inf. Process. Syst., pp. 4436-4446. Curran Associates, Inc. (2018).

[8] J. C. Snyder, M. Rupp, K. Hansen, K.-R. Müller and K. Burke, Finding density functionals with machine learning, Phys. Rev. Lett. 108(25), 253002 (2012), doi:10.1103/PhysRevLett.108.253002.

[9] L. Li, J. C. Snyder, I. M. Pelaschier, J. Huang, U.-N. Niranjan, P. Duncan, M. Rupp, K.-R. Müller and K. Burke, Understanding machine-learned density functionals, Int. J. Quantum Chem. 116(11), 819 (2016), doi:10.1002/qua.25040.

[10] F. Brockherde, L. Vogt, L. Li, M. E. Tuckerman, K. Burke and K.-R. Müller, Bypassing the Kohn-Sham equations with machine learning, Nat. Commun. 8(1), 872 (2017), doi:10.1038/s41467-017-00839-3.

[11] K. Ryczko, D. A. Strubbe and I. Tamblyn, Deep learning and density-functional theory, Phys. Rev. A 100(2), 022512 (2019), doi:10.1103/PhysRevA.100.022512.

[12] J. R. Moreno, G. Carleo and A. Georges, Deep learning the hohenbergkohn maps of density functional theory, Phys. Rev. Lett. 125, 076402 (2020), doi:10.1103/PhysRevLett.125.076402.

[13] C. A. Custódio, É. R. Filletti and V. V. França, Artificial neural networks for density-functional optimizations in fermionic systems, Sci. Rep. 9(1), 1886 (2019), doi:10.1038/s41598-018-37999-1. 
[14] K. Hansen, G. Montavon, F. Biegler, S. Fazli, M. Rupp, M. Scheffler, O. A. Von Lilienfeld, A. Tkatchenko and K.-R. Müller, Assessment and validation of machine learning methods for predicting molecular atomization energies, J. Chem. Theory Comput. 9(8), 3404 (2013), doi:10.1021/ct400195d.

[15] K. T. Schütt, H. Glawe, F. Brockherde, A. Sanna, K. R. Müller and E. K. U. Gross, How to represent crystal structures for machine learning: Towards fast prediction of electronic properties, Phys. Rev. B 89(20), 205118 (2014), doi:10.1103/PhysRevB.89.205118.

[16] K. Hansen, F. Biegler, R. Ramakrishnan, W. Pronobis, O. A. Von Lilienfeld, K.-R. Müller and A. Tkatchenko, Machine learning predictions of molecular properties: Accurate many-body potentials and nonlocality in chemical space, J. Phys. Chem. Lett. 6(12), 2326 (2015), doi:10.1021/acs.jpclett.5b00831.

[17] P. J. Ballester and J. B. Mitchell, A machine learning approach to predicting proteinligand binding affinity with applications to molecular docking, Bioinformatics 26(9), 1169 (2010), doi:10.1093/bioinformatics/btq112.

[18] M. A. Khamis, W. Gomaa and W. F. Ahmed, Machine learning in computational docking, Artif. Intell. Med. 63(3), 135 (2015), doi:10.1016/j.artmed.2015.02.002.

[19] J. Jiménez, M. Skalic, G. Martinez-Rosell and G. De Fabritiis, K deep: Protein-ligand absolute binding affinity prediction via 3d-convolutional neural networks, J. Chem. Inf. Model. 58(2), 287 (2018), doi:10.1021/acs.jcim.7b00650.

[20] K.-I. Funahashi, On the approximate realization of continuous mappings by neural networks, Neural Netw. 2(3), 183 (1989), doi:10.1016/0893-6080(89)90003-8.

[21] R. Caruana, Multitask learning, Mach. Learn. 28(1), 41 (1997), doi:10.1023/A:1007379606734.

[22] J. Behler, Perspective: Machine learning potentials for atomistic simulations, J. Chem. Phys. 145(17), 170901 (2016), doi:10.1063/1.4966192.

[23] K. Mills, K. Ryczko, I. Luchak, A. Domurad, C. Beeler and I. Tamblyn, Extensive deep neural networks for transferring small scale learning to large scale systems, Chem. Sci. 10(15), 4129 (2019), doi:10.1039/C8SC04578J.

[24] H. Jung, S. Stocker, C. Kunkel, H. Oberhofer, B. Han, K. Reuter and J. T. Margraf, Size-extensive molecular machine learning with global representations, Chem Systems Chem 2, e1900052 (2020), doi:10.1002/syst.201900052.

[25] N. Saraceni, S. Cantori and S. Pilati, Scalable neural networks for the efficient learning of disordered quantum systems, Phys. Rev. E 102, 033301 (2020), doi:10.1103/PhysRevE.102.033301.

[26] F. A. Faber, L. Hutchison, B. Huang, J. Gilmer, S. S. Schoenholz, G. E. Dahl, O. Vinyals, S. Kearnes, P. F. Riley and O. A. Von Lilienfeld, Prediction errors of molecular machine learning models lower than hybrid dft error, J. of Chem. Theory Comput. 13(11), 5255 (2017), doi:10.1021/acs.jctc.7b00577. 
[27] A. Aspect and M. Inguscio, Anderson localization of ultracold atoms, Phys. Today 62(8), 30 (2009), doi:10.1063/1.3206092.

[28] M. P. Fisher, P. B. Weichman, G. Grinstein and D. S. Fisher, Boson localization and the superfluid-insulator transition, Phys. Rev. B 40(1), 546 (1989), doi:10.1103/PhysRevB.40.546.

[29] P. Mujal, A. Polls, S. Pilati and B. Juliá-Díaz, Few-boson localization in a continuum with speckle disorder, Phys. Rev. A 100, 013603 (2019), doi:10.1103/PhysRevA.100.013603.

[30] G. Roati, C. D'Errico, L. Fallani, M. Fattori, C. Fort, M. Zaccanti, G. Modugno, M. Modugno and M. Inguscio, Anderson localization of a non-interacting Bose-Einstein condensate, Nature 453(7197), 895 (2008), doi:10.1038/nature07071.

[31] J. Billy, V. Josse, Z. Zuo, A. Bernard, B. Hambrecht, P. Lugan, D. Clément, L. SanchezPalencia, P. Bouyer and A. Aspect, Direct observation of Anderson localization of matter waves in a controlled disorder, Nature 453(7197), 891 (2008), doi:10.1038/nature07000.

[32] J. Huntley, Speckle photography fringe analysis: assessment of current algorithms, Appl. Opt. 28(20), 4316 (1989), doi:10.1364/AO.28.004316.

[33] M. Modugno, Collective dynamics and expansion of a bose-einstein condensate in a random potential, Phys. Rev. A 73, 013606 (2006), doi:10.1103/PhysRevA.73.013606.

[34] M. Płodzień, D. Wiater, A. Chrostowski and T. Sowiński, Numerically exact approach to few-body problems far from a perturbative regime (2018), arXiv:1803.08387.

[35] P. Mujal, Interacting ultracold few-boson systems, Ph.D. thesis, Universitat de Barcelona (2019).

[36] P. Mujal, A. Martínez Miguel, A. Polls, B. Juliá-Díaz and S. Pilati, Database used in the analysis, Zenodo (2020), doi:10.5281/zenodo.4058492.

[37] M. A. Nielsen, Neural Networks and Deep Learning, Determination Press (2015).

[38] D. P. Kingma and J. Ba, Adam: A method for stochastic optimization (2014), arXiv:1412.6980.

[39] F. Chollet et al., Keras, https://keras.io (2015).

[40] K.-R. Müller, M. Finke, N. Murata, K. Schulten and S.-i. Amari, A numerical study on learning curves in stochastic multilayer feedforward networks, Neural Computation 8(5), 1085 (1996), doi:10.1162/neco.1996.8.5.1085

[41] B. Huang and O. A. von Lilienfeld, Communication: Understanding molecular representations in machine learning: The role of uniqueness and target similarity, J. Chem. Phys. 145(16), 161102 (2016), doi:10.1063/1.4964627.

[42] K. He, X. Zhang, S. Ren and J. Sun, Deep residual learning for image recognition, In Proc. IEEE Int. Conf. Comput. Vis., pp. 770-778, doi:10.1109/CVPR.2016.90 (2016).

[43] K. Simonyan and A. Zisserman, Very deep convolutional networks for large-scale image recognition (2014), arXiv:1409.1556. 